\title{
Absorbable Implant to Treat Nasal Valve Collapse
}

\author{
Marion San Nicoló, MD ${ }^{1}$ Klaus Stelter, MD ${ }^{2}$ Haneen Sadick, MD ${ }^{3}$ Murat Bas, MD \\ Alexander Berghaus, MD ${ }^{1}$
}

${ }^{1}$ Department of Otorhinolaryngology, Head and Neck Surgery, Ludwig Maximilian University of Munich, Munich, Germany

2 HNO Zentrum Mangfall-Inn, Rosenheim, Germany

3 Division of Facial Plastic Surgery, Department of

Otorhinolaryngology, University of Mannheim, Mannheim, Germany

${ }^{4}$ Department of Otorhinolaryngology, Head and Neck Surgery, Klinikum rechts der Isar, Technical University Munich, Munich, Germany

\begin{abstract}
Address for correspondence Marion San Nicoló, MD, Department of Otorhinolaryngology, Head and Neck Surgery, Ludwig Maximilian University of Munich, Marchioninistr. 15, Munich 81377, Germany (e-mail: marion.sannicolo@med.uni-muenchen.de).
\end{abstract}

\begin{abstract}
Keywords

- nasal valve

- implant

- rhinoplasty

- lateral wall insufficiency

Objective To evaluate the safety and effectiveness of an absorbable implant for lateral cartilage support in subjects with nasal valve collapse (NVC) with 12 months follow-up. Methods Thirty subjects with Nasal Obstruction Symptom Evaluation (NOSE) score $\geq 55$ and isolated NVC were treated; 14 cases were performed in an operating suite under general anesthesia and 16 cases were performed in a clinic-based setting under local anesthesia. The implant, a polylactic acid copolymer, was placed with a delivery tool within the nasal wall to provide lateral cartilage support. Subjects were followed up through 12 months postprocedure.

Results Fifty-six implants were placed in 30 subjects. The mean preoperative NOSE score was $76.7 \pm 14.8$, with a range of 55 to 100 . At 12 months, the mean score was $35.2 \pm 29.2$, reflecting an average within-patient reduction of $-40.9 \pm 31.2$ points. The majority (76\%) of the subjects were responders defined as having at least one NOSE class improvement or a NOSE score reduction of at least $20 \%$. There were no adverse changes in cosmetic appearance at 12 months postprocedure. Three implants in three subjects required retrieval within 30 days postprocedure and resulted in no clinical sequelae.

Conclusion This study demonstrates safety and effectiveness of an absorbable implant for lateral cartilage support in subjects with NVC at 12 months postprocedure.
\end{abstract}

The nasal valve, first described in the early 20th century by Mink, ${ }^{1}$ is a complex, three-dimensional, dynamically alternating structure that controls nasal airflow resistance. A dysfunction of the nasal valve can lead to nasal obstruction and a significant drop in the quality of life (QOL) for patients. ${ }^{2}$ As defined by the Hagen-Poiseuille law, the flow through a tube is proportional to the fourth power of the radius of the tube and inversely proportional to the pressure difference across the tube. Thus, even a small decrease in the valve area can contribute to severe nasal obstruction.

The nasal valve collapse(NVC)can bestatic ordynamic.Static NVC consists of an anatomically narrowed nasal valve region, which causes obstruction. Dynamic NVC is caused by insuffi- cient cartilaginous support of the lateral nasal wall, leading to lateral wall insufficiency. ${ }^{3,4}$ Common causes of NVC are prior rhinoplasty, aging, nasal trauma, and congenital abnormalities. Therapies to correct NVC include invasive surgical procedures and nonsurgical solutions to temporarily dilate the nasal valve, such as Breathe Right strips or nasal cones. Surgical strategies that involve septoplasty ${ }^{5}$ or inferior turbinate reduction ${ }^{6}$ may reduce negative inspiratory pressure by enlarging the airway, but these procedures do not directly address weakness in the lateral wall. Procedures intended to address the weakness of the lateral wall include cartilaginous grafts, typically harvested from the nasal septum, ${ }^{7}$ ear ${ }^{8}$ or rib cartilage ${ }^{9}$ that can be placed as lateral crural strut grafts, ${ }^{10}$ alar batten grafts, ${ }^{11}$ or butterfly
DOI http://dx.doi.org/ 10.1055/s-0037-1598655. ISSN 0736-6825.
Copyright (c) 2018 by Thieme Medical Publishers, Inc., 333 Seventh Avenue, New York, NY 10001, USA. Tel: +1(212) 584-4662.
License terms

()(1) 
grafts. ${ }^{12}$ Implants made from nonabsorbable alloplastic materials have also been used for treatment of NVC including expanded polytetrafluoroethylene ${ }^{13}$ and high-density porous polyethylene. ${ }^{14,15}$ These nonabsorbable materials have not gained wide use as they require invasive surgical procedures and are associated with increased risks of infection, extrusion, and the potential need for revision procedures.

While surgery to strengthen the lateral wall has been shown to significantly improve the QOL for subjects suffering from nasal airway obstruction, current procedures can be invasive and have the potential to permanently alter the patient's appearance. ${ }^{16}$ In this study, a minimally invasive technique to address NVC by supporting the weakened nasal lateral wall cartilage with an absorbable implant is described.

\section{Methods}

\section{Study Design}

This prospective, single cohort, nonrandomized study evaluating the safety and effectiveness of an absorbable nasal implant (Spirox Inc., Menlo Park, CA) enrolled 30 subjects at three investigational sites in Germany.

Consecutive subjects at each site were screened for potential enrollment. Eligible subjects were invited to participate in the study. The baseline visit included a medical history review, an evaluation of symptoms, an assessment of nasal airway obstruction, and photodocumentation of nasal appearance. Demographic information, such as age, gender, and date of onset of nasal obstruction, was collected. History of any prior nasal trauma, surgery, and other medical conditions were noted. Physical examination including anterior rhinoscopy and nasal endoscopy were performed to determine the degree to which NVC contributed to the overall nasal airway obstruction. The degree of nasal obstruction was rated by investigators on a severity scale as none, mild, moderate, or severe.

Subjects were treated under general or local anesthesia. No concomitant nasal procedures were performed. Follow-up visits took place at week 1 and months 1, 3, 6, and 12 postprocedure. Internal and external nasal examinations were performed ateach visit, as well as Nasal Obstruction Symptom Evaluation (NOSE) score collection, ${ }^{17}$ pain assessments, presence of a foreign body sensation, and assessment of cosmetic changes. Physical examinations included an evaluation of nasal skin and nasal mucosa appearance, and the presence of any implant extrusions, fractures, or migration. Cosmetic changes were assessed using four photographicviewsobtained underbothstaticand fullinhalation conditions (frontal view, left side, right side, and chin up). An independent physician assessed cosmetic changes by comparing baseline images to the follow-up images and categorizing the comparisons as no change, significantly better, or significantly worse. For example, significantly better changes included structural changes such as widening of the nasal vault, whereas significantly worse included a narrowing of the nasal vault.

\section{Subjects}

Eligible subjects were adults with NVC identified as the primary contributor to nasal obstruction with a NOSE score $\geq 55$ at baseline. Subjects were ineligible if they had septoplasty or turbinate reduction procedures within 6 months or rhinoplasty procedures within 12 months prior to the planned index procedure. Additional exclusion criteria were recurrent nasal infections, intranasal steroid treatment 2 weeks prior or planned for 2 weeks postindex procedure, permanent nasal implants or dilators, a history of (pre) cancerous or cancerous lesions, and/or radiation exposure or chemotherapy within 24 months of the study. Subjects with bleeding disorders, those with significant systemic diseases, or those requiring nasal oxygen or continuous positive airway pressure (CPAP) were not eligible to participate.

\section{Implant and Delivery Tool}

The absorbable nasal implant comprises a 70:30 blend of poly(L-lactide) and poly(D-lactide). It is introduced through an endonasal insertion technique using a delivery tool. The implant is primarily a ribbed cylindrical structure with an apical forked end. The implant is designed to provide support to the upper and lower lateral nasal cartilages. The geometry of the forked end is flexible and collapses to fit within the 16gauge cannula portion of the delivery tool prior to placement. The forked end first exits the delivery tool cannula and expands open as the implant is deployed into the tissue. This fork deployment is designed to anchor the implant in place during the acute implantation step.

Once delivered, the apical, forked end of the implant is positioned over and adjacent to the frontal process of the maxilla while the main body extends caudally toward the alar region. The implant is flexible and therefore has the ability to conform to the natural curvature of the lateral wall plane. In this location, the implant is adjacent to the upper and lower lateral cartilages to provide support and strengthen the lateral wall.

\section{Implantation Technique}

Implantation steps are illustrated in -Fig. 1. Preprocedure, in each subject, the nasal anatomy as well as the area of maximum lateral wall collapse during inspiration were examined and marked to identify the target implant location and cannula insertion trajectory. The area of maximum collapse was evaluated using the modified Cottle maneuver. The target implant location was established to position the forked tip of the implant adjacent and across the maxilla bone to provide cantilever support, and the main cylindrical body of the implant was positioned along a trajectory to support the upper and lower lateral cartilages crossing the area of maximum collapse. In cases where the collapse was too lateral for placement of the implant, the implant was positioned as close as possible to the area of collapse (-Fig. 1A). Although this positioning does not directly cross over the area of the collapse, the implant should provide sufficient lateral wall support.

After subject marking, the implant was loaded into the delivery toolcannulaand wasintroduced through thevestibular skin using an intranasal entry point close to the alar rim (-Fig. 1B). Care was taken to ensure that the cannula at the 


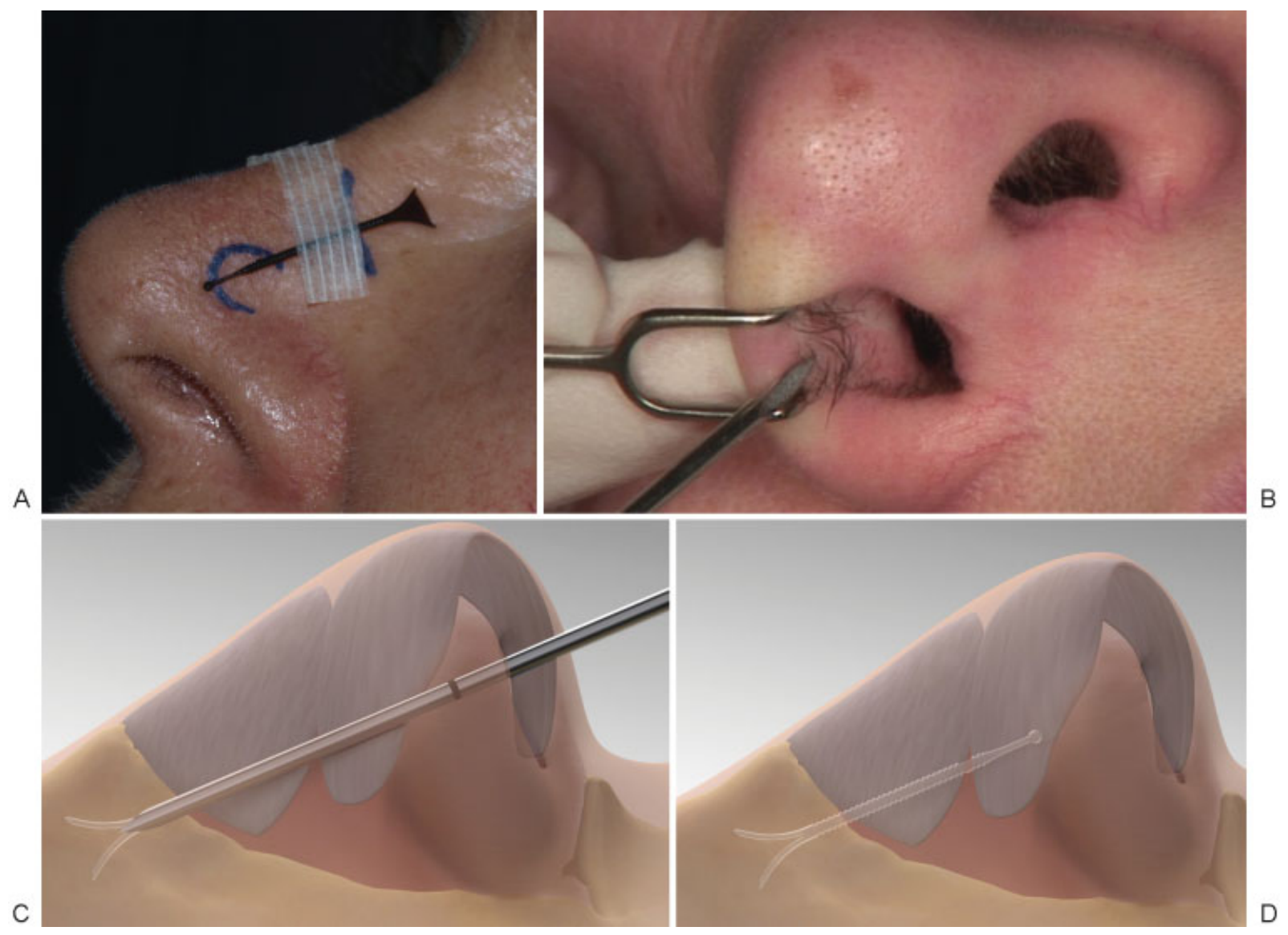

Fig. 1 Implantation technique. (A) Planned location of the implant relative to the area of collapse. (B) Placement of pierce point for delivery tool placement. (C) Delivery tool placement. (D) Delivery tool removal.

entrypointdid not penetrate through thelowerlateralcartilage. The cannula was then advanced over the lateral surface of the lower lateral cartilage and over the upper lateral cartilage to the frontal process of the maxilla. From there, it was advanced over the maxilla to a point where the apical portion of the implant would be positioned over the maxilla while the main cylindrical portion is positioned in the lateral wall ( - Fig. 1C). The implant was then deployed, and the delivery tool was retracted and removed, leaving the implant in place to support the upper and lower lateral cartilages ( - Fig. 1D).

\section{Statistical Analysis}

The NOSE scale is a validated disease-specific QOL instrument. ${ }^{17}$ It uses a 20 -point scale to capture breathing symptoms, with higher scores indicating more severe symptoms than lower scores. NOSE score results are converted to a 100point scale by multiplying the total score by 5 . This analysis includes the change in NOSE scores from baseline (preoperative) to 3,6 , and 12 months. A paired $t$-test was used to determine whether the mean at follow-up time points was significantly different from the preoperative mean while controlling for within-subject correlation. A sensitivity analyses using a mixed model for repeated measures (MMRM) including baseline score as a fixed covariate was performed for comparison.
A NOSE score severity classification system was developed by Lipan and Most based on the data from 345 patients with and without nasal airway obstruction. ${ }^{18}$ Their analysis derived clinically relevant severity classes of NOSE scores: mild (5-25 points), moderate (30-50 points), severe (55-75 points), or extreme (80-100 points). The analysis reported herein used this classification system to report the percentage of subjects in each category at baseline, 3, 6, and 12 months as well as to classify subjects as responders or nonresponders to the procedure. Responders are defined as subjects that have at least one NOSE class improvement or a NOSE score reduction of at least $20 \%$.

Statistical analyses were performed by an independent statistician (Axio Research, Seattle, WA) using SAS version 9.4 and $\mathrm{R}$ version 3.2.3.

\section{Results}

Subject demographics and baseline disease characteristics are provided in -Table 1. A significant percentage of the subjects had previous surgeries (66\%); all subjects had confirmed NVC as the primary contributor to nasal airway obstruction. A total of 56 implants were placed in 30 subjects. Fourteen procedures took place in an operating suite under general anesthesia, and 16 procedures were conducted in a 
236 Absorbable Implant to Treat NVC San Nicoló et al.

Table 1 Subject demographics and medical history

\begin{tabular}{|c|c|c|}
\hline Attribute & & $\begin{array}{l}\text { Result } \\
(N=30)\end{array}$ \\
\hline \multirow[t]{2}{*}{ Gender } & Female & $12(40 \%)$ \\
\hline & Male & $18(60 \%)$ \\
\hline \multirow[t]{3}{*}{ Age $(y)$} & Mean \pm SD & $51.1 \pm 14.5$ \\
\hline & Minimum & 24 \\
\hline & Maximum & 77 \\
\hline \multirow[t]{3}{*}{ BMI $\left(\mathrm{kg} / \mathrm{m}^{2}\right)$} & Mean \pm SD & $27.6 \pm 5.3$ \\
\hline & Minimum & 22 \\
\hline & Maximum & 44 \\
\hline \multirow[t]{3}{*}{ Race (n (\%)) } & Asian & $1(3.3 \%)$ \\
\hline & White & $28(93.4 \%)$ \\
\hline & Black & $1(3.3 \%)$ \\
\hline \multirow[t]{3}{*}{ Baseline NOSE score } & Mean \pm SD & $76.7 \pm 14.8$ \\
\hline & Minimum & 55 \\
\hline & Maximum & 100 \\
\hline \multirow[t]{19}{*}{ Prior history } & \multicolumn{2}{|l|}{ Nasal trauma } \\
\hline & Yes & $8(26.7 \%)$ \\
\hline & No & $22(73.3 \%)$ \\
\hline & \multicolumn{2}{|l|}{ Nasal surgery } \\
\hline & Yes & $19(63.3 \%)$ \\
\hline & No & $11(36.7 \%)$ \\
\hline & \multicolumn{2}{|l|}{ Years since most recent nasal surgery } \\
\hline & $<1 \mathrm{y}^{\mathrm{a}}$ & $3(15.8 \%)$ \\
\hline & $1-3 y$ & $8(42.1 \%)$ \\
\hline & $3-5 y$ & $1(5.3 \%)$ \\
\hline & $>5 y$ & $7(36.8 \%)$ \\
\hline & \multicolumn{2}{|l|}{ Nonsurgical nasal treatments } \\
\hline & Yes & 5 (16.7\%) \\
\hline & No & $25(83.3 \%)$ \\
\hline & \multicolumn{2}{|l|}{ Medications } \\
\hline & None & $10(33.3 \%)$ \\
\hline & Topical steroid use & $1(3.3 \%)$ \\
\hline & External nasal dilator & $4(13.3 \%)$ \\
\hline & Other medications & $15(50 \%)$ \\
\hline \multirow[t]{5}{*}{ Nasal endoscopy findings } & None & $15(50 \%)$ \\
\hline & Deviated septum & $13(43.3 \%)$ \\
\hline & Inferior turbinate hypertrophy & $2(6.7 \%)$ \\
\hline & Middle meatus pathology & $0(0.0 \%)$ \\
\hline & Choanae and nasopharynx pathology & $0(0.0 \%)$ \\
\hline \multirow[t]{2}{*}{ Degree of nasal obstruction } & Moderate & $17(56.7 \%)$ \\
\hline & Severe & $13(43.3 \%)$ \\
\hline \multirow{2}{*}{$\begin{array}{l}\text { NVC primary contributor to nasal } \\
\text { obstruction }\end{array}$} & Yes & $30(100 \%)$ \\
\hline & No & $0(0.0 \%)$ \\
\hline
\end{tabular}

Abbreviations: BMI, body mass index; NOSE, Nasal Obstruction Symptom Evaluation; NVC, nasal valve collapse; SD, standard deviation.

${ }^{a}$ Does not include rhinoplasty or nasal valve surgery per protocol. 
Table 2 Nasal examination cumulative observations through 12 months follow-up

\begin{tabular}{|c|c|}
\hline Attribute & $(N=30)$ \\
\hline \multicolumn{2}{|c|}{ External nasal examination } \\
\hline \multicolumn{2}{|l|}{ Implant extrusion } \\
\hline No & $30(100 \%)$ \\
\hline \multicolumn{2}{|l|}{ Skin appearance } \\
\hline Normal & $28(93.3 \%)$ \\
\hline Hematoma & $1(3.3 \%)$ \\
\hline Inflammation & $1(3.3 \%)$ \\
\hline \multicolumn{2}{|c|}{ Internal nasal examination } \\
\hline \multicolumn{2}{|l|}{ Implant retrieval } \\
\hline Yes & $3(10 \%)$ \\
\hline No & $27(90 \%)$ \\
\hline \multicolumn{2}{|c|}{ Mucosal appearance } \\
\hline Normal & $28(93.3 \%)$ \\
\hline Inflammation & $1(3.3 \%)^{\mathrm{a}}$ \\
\hline Infection & $1(3.3 \%)^{a}$ \\
\hline \multicolumn{2}{|c|}{ Implant break/fracture } \\
\hline No & $30(100 \%)$ \\
\hline \multicolumn{2}{|l|}{ Implant migration } \\
\hline No & $30(100 \%)$ \\
\hline
\end{tabular}

${ }^{\mathrm{a}}$ Not device procedure related.

clinic-based setting with local anesthesia. Bilateral, single implants were placed in 26 subjects, and a unilateral, single implant was placed in 4 subjects. Implants were successfully delivered during the initial attempt in $91 \%$ of the cases. All procedures resulted in successful placement of implant(s) to the target location(s). No device-related adverse events were reported during the index procedure.

- Table 2 summarizes cumulative internal and external nasal examination results through 12 months of follow-up. There was no evidence of transcutaneous extrusions. The skin examination was normal with two exceptions: one patient with a hematoma was noted at the 1 week followup time point, and one patient with inflammation was noted at the 1-month follow-up examination. Both observations resolved prior to the subsequent follow-up examination. Internal nasal examinations were normal across all time points with the two exceptions: one report of nondevicerelated inflammation was noted at 1 week, and one nondevice-related infection was reported at 6-month follow-up (rhinitis). There was no evidence of implant fracture and no findings of implant migration based on internal and external physical examination.

Three subjects required retrievals of a single implant within the 1-month follow-up period. These events were attributed to the implantation technique in two instances and possible nasal manipulation by the subject in one instance. The two retrievals related to the implantation technique were attributed to an incomplete delivery, resulting in the tip of the caudal end of the implant lying very close to the cannula entry point causing subsequent exposure. Using forceps, the investigator was able to remove the entire implant without difficulty and without the need for anesthesia. The third retrieval occurred when a tip of the implant protruding through the implantation site on one side was observed and retrieved by the investigator. In this case, the subject reported to have blown his nose forcibly, multiple times during the week following the procedure. There was no erythema, bleeding, swelling, or pain. The protruding portion of the implant appeared to be mobile and was removed easily with forceps and without anesthetic. During the nasal endoscopic examination, it was noted that there was no sign of infection or a lesion due to the remaining upper part of the implant on the lateral nasal wall. These three devicerelated events resolved with no clinical sequelae.

Follow-up outcomes including pain assessments, evaluation of foreign body sensation, the independent assessment of cosmetic change, and adverse events at $1,3,6$, and 12 months are summarized in - Table 3. There were no reports of moderate or severe pain at $1,3,6$, or 12 months. Four subjects reported a "mild" foreign body sensation at month 1 , and three subjects at month 12 . Independent physician photography review reported one subject with an adverse cosmetic change at 3 months postprocedure that subsequently resolved. At 6 and 12 months, there were no adverse cosmetic changes identified, and three subjects were classified as having significant cosmetic improvements at 12 months, where the independent reviewer observed less alar retraction for 2 subjects, and 1 subject where the nasal vaults were more open bilaterally. Approximately half of the subjects wore eyeglasses throughout the follow-up period. There were five adverse event related to the study device/ procedure that occurred within the 1-month postprocedure follow-up. No subsequent device/procedure related adverse event was observed.

Within-subject changes in NOSE score from baseline to 3, 6, and 12 months are summarized in - Table 4. The mean preoperative NOSE score was $76.7 \pm 14.8$. Twelve months postprocedure, the mean NOSE score was $35.2 \pm 29.2$, reflecting an average within-subject reduction of $-40.9 \pm 31.2$ points. The paired $t$-test showed significant differences between the mean baseline and follow-up NOSE score at all three follow-up time-points ( $p<0.001$ for months 3,6 , and 12 ). MMRM results were similar. - Table 5 presents the response rates at 3, 6, and 12 months. The majority (76\%) of subjects were classified as responders at 12 months, demonstrating sustained reductions in nasal obstruction symptoms. -Fig. 2 presents NOSE score categories preprocedure and at 12 months in terms of the number of subjects in each NOSE severity class. Preprocedure, all subjects were classified as extreme or severe. At 12 months, $66 \%$ of the subjects were classified as mild or moderate, and the number of subjects classified as extreme and severe was reduced to 3 and $31 \%$, respectively. 
Table 3 Device tolerability, cosmetic changes, and adverse events at 1, 3, 6, and 12 months

\begin{tabular}{|c|c|c|c|c|}
\hline \multirow[t]{2}{*}{ Attribute } & 1 mo postprocedure & 3 mo postprocedure & 6 mo postprocedure & 12 mo postprocedure \\
\hline & $(N=30)$ & $(N=29)$ & $(N=30)$ & $(N=29)$ \\
\hline \multicolumn{5}{|l|}{ Pain assessment } \\
\hline None/mild & $30(100 \%)$ & $29(100 \%)$ & $29(96.7 \%)$ & $29(100 \%)$ \\
\hline Moderate/severe & $0(0.0 \%)$ & $0(0.0 \%)$ & $0(0.0 \%)$ & $0(0.0 \%)$ \\
\hline Not assessed & $0(0.0 \%)$ & $0(0.0 \%)$ & $1(3.3 \%)$ & $0(0.0 \%)$ \\
\hline \multicolumn{5}{|l|}{ Foreign body sensation } \\
\hline None & $26(86.7 \%)$ & $27(93.1 \%)$ & $27(90.0 \%)$ & $26(89.7 \%)$ \\
\hline Mild & $4(13.3 \%)$ & $2(6.9 \%)$ & $3(10 \%)$ & $3(10.3 \%)$ \\
\hline Photography review (cosmetic change from baseline) & $(N=30)$ & $(N=29)$ & $(N=27)$ & $(N=27)$ \\
\hline None & $28(93.3 \%)$ & $25(86.2 \%)$ & $24(88.9 \%)$ & 24 (88.9\%) \\
\hline Yes-insignificant & $0(0.0 \%)$ & $0(0.0 \%)$ & $0(0.0 \%)$ & $0(0.0 \%)$ \\
\hline Yes-significant-worse & $0(0.0 \%)$ & $1(3.4 \%)$ & $0(0.0 \%)$ & $0(0.0 \%)$ \\
\hline Yes-significant-better & $2(6.7 \%)$ & $3(10.3 \%)$ & $3(11.1 \%)$ & $3(11.1 \%)$ \\
\hline \multicolumn{5}{|l|}{ Adverse events } \\
\hline Device related $^{\mathrm{a}}$ & 5 & & & \\
\hline Other $^{\mathrm{b}}$ & 4 & 4 & 2 & - \\
\hline
\end{tabular}

${ }^{a}$ Device-related adverse events include three device retrievals, one hematoma, and one inflammation. ${ }^{\mathrm{b} O t h e r}$ nondevice-/procedure-related adverse events includes rhinitis, common colds, vertigo, rhinorrhea, acute hypertension, headache, hernia repair, and epistaxis.

Table 4 Pre- and postprocedure NOSE scores and change from baseline value

\begin{tabular}{|c|c|c|c|c|c|c|c|}
\hline \multirow[t]{2}{*}{ Statistics } & \multirow{2}{*}{$\begin{array}{l}\text { Baseline } \\
\text { NOSE score }\end{array}$} & \multicolumn{2}{|c|}{3 mo postprocedure } & \multicolumn{2}{|c|}{6 mo postprocedure } & \multicolumn{2}{|c|}{12 mo postprocedure } \\
\hline & & NOSE score & $\begin{array}{l}\text { Change from } \\
\text { baseline }\end{array}$ & NOSE score & $\begin{array}{l}\text { Change from } \\
\text { baseline }\end{array}$ & NOSE score & $\begin{array}{l}\text { Change from } \\
\text { baseline }\end{array}$ \\
\hline$N$ & 30 & 29 & 29 & 30 & 30 & 29 & 29 \\
\hline Mean & 76.7 & 28.4 & -48.4 & 33.3 & -43.3 & 35.2 & -40.9 \\
\hline SD & 14.8 & 26.9 & 27.8 & 29.7 & 31.3 & 29.2 & 31.2 \\
\hline Median & 75 & 20 & -50 & 27.5 & -45 & 35 & -45 \\
\hline$p$-Value ${ }^{a}$ & & & $<0.001$ & & $<0.001$ & & $<0.001$ \\
\hline
\end{tabular}

Abbreviations: NOSE, Nasal Obstruction Symptom Evaluation; SD, standard deviation.

${ }^{a} p$-Values are from paired $t$-tests comparing the mean preoperative NOSE score to the mean score at each follow-up time point.

Table 5 Response rate ${ }^{a}$ at 3, 6, and 12 months after procedure

\begin{tabular}{|l|l|l|}
\hline & $N$ & Responders, $^{\text {b }} N(\%)$ \\
\hline 3 mo postprocedure & 29 & $25(86.2 \%)$ \\
\hline 6 mo postprocedure & 30 & $24(80 \%)$ \\
\hline 12 mo postprocedure & 29 & $22(75.9 \%)$ \\
\hline
\end{tabular}

aResponse rate is based on the number of subjects with data at each visit. ${ }^{\mathrm{b}}$ Responders are subjects with an improvement of at least one NOSE score category or a $20 \%$ reduction in NOSE score.

\section{Discussion}

Nasal airway obstruction can be caused by a combination of anatomical and structural abnormalities, including a weakened lateral nasal wall that can result in NVC.
Data presented herein describe the first-in-human experience characterizing the use of a novel absorbable implant to support the upper and lower lateral cartilages in subjects with NVC through 12 months. Subjects noted a significant reduction in nasal obstruction symptoms after the procedure through 12 months. The strengths of this study are of a disease-specific instrument for treatment of nasal obstruction due to NVC, long-term follow-up, and prospective patient evaluation.

Recently, a meta-analysis was conducted by Rhee et $\mathrm{al}^{19}$ of studies covering conventional invasive surgical procedures such as septoplasty, turbinate reduction, and functional rhinoplasty, in combination or alone for treatment of nasal airway obstruction. The analysis showed a weighted mean pretreatment NOSE score of 65 points and a weighted mean posttreatment NOSE score of 23 points, resulting in an improvement from baseline of 42 points. In this study, 


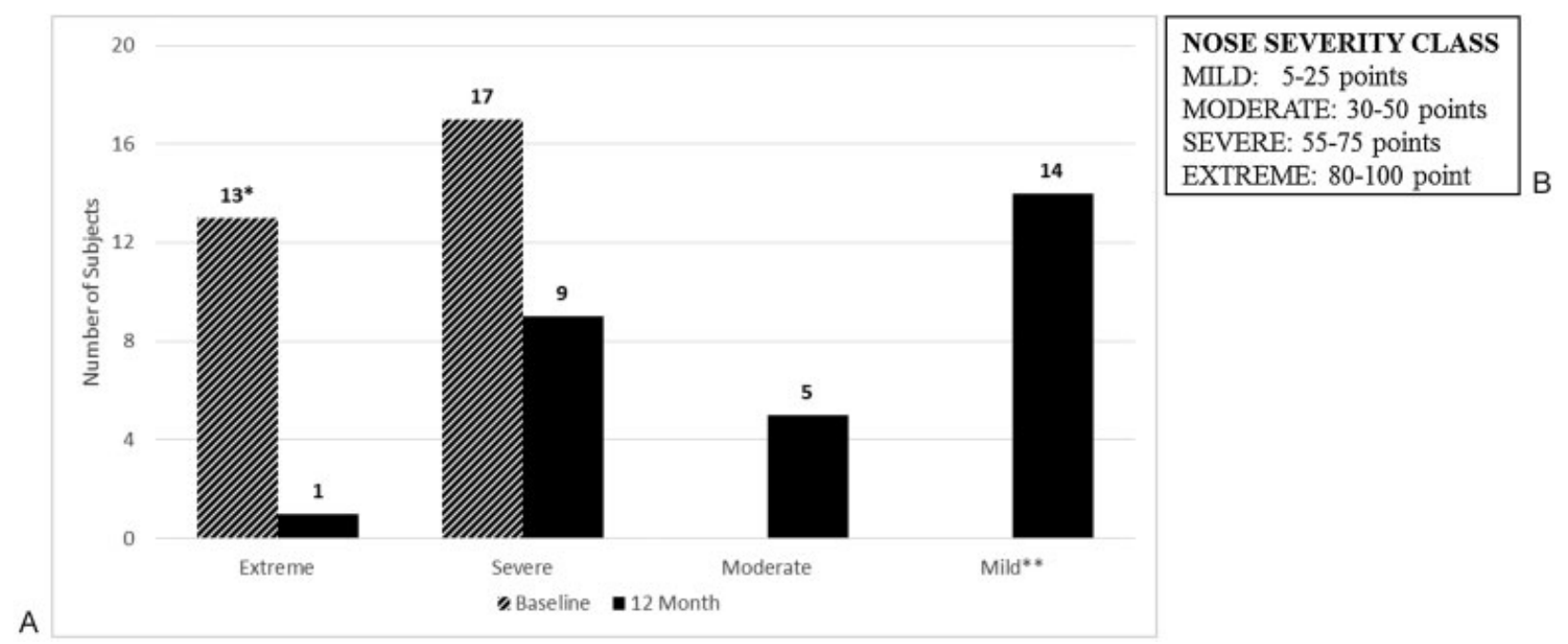

Fig. 2 Nasal Obstruction Symptom Evaluation (NOSE) severity class at baseline and 12 months postprocedure. *One subject who was classified as extreme at baseline did not complete the 12-month NOSE score assessment. ${ }^{* *}$ Four subjects at 12 -month follow-up had a NOSE score of 0 that have been categorized as mild for this analysis.

subjects with NVC as a primary contributor to their nasal airway obstruction symptoms were treated in stand-alone procedures. The results from this study are within the range of the meta-analysis findings with a mean improvement in NOSE score of 41 points at 12 months. In addition, this study showed $76 \%$ of the subjects were classified as responders defined as having at least one NOSE class improvement or a NOSE score reduction of at least $20 \%$.

The implant created no adverse cosmetic change as confirmed by the independent photographic review. Spreader grafts and batten grafts may lead to changes in the external appearance of the nose, including widening of the middle third of the nose, blunting of the alar crease, and widening of the nasal tip. ${ }^{16}$ However, many of these grafting procedures are more extensive (i.e., require cartilage repair or replacement) than the procedures indicated for the subject implant (i.e., cartilage support only). The lack of significant cosmetic changes in this study may be attributable to the design and position of the implant; the low profile of the implant, particularly in the region that aligns with the thinner skin above the maxillary transition, allows for minimal change in the external nasal appearance. The presence of the implant in the nasal wall was well-tolerated by the subjects as evidenced by minimal pain scores and minimal foreign body sensation to the device's presence in the nose. The implant also did not interfere with the use of eye glasses.

There were a total of five device-related adverse events reported in four subjects. These events included one case of hematoma, one case of inflammation, and three of implant retrievals. All events resolved with no clinical sequelae. Investigators concluded that the three implant retrievals were the result of suboptimal implantation method during initial cases or possibly significant patient nasal manipulation during the first postoperative week. There was no evidence of adverse physiological tissue rejection, infection, and/or significant implant migrations in contrast with the extrusion events reported in the literature for more invasive procedures involving permanent, nonabsorbable allografts. ${ }^{13,14}$

The efficacy of the implant in reducing nasal obstruction is demonstrated by a significant reduction in NOSE scores through 12 months of follow-up, the gold standard for rhinoplasty studies. This current study represents a first-inman demonstration of a new technique, as it has a modest sample size, a nonrandomized study design, and a heterogeneous subject population with respect to prior history of surgery. Future studies with a lager patient population should address this as well as to include comparisons of the efficacy, morbidity, and cost-effectiveness of this technique to standard surgical techniques and placebo treatments.

The absorbable nasal implant used in this study comprised 70\% poly(L-lactide) and 30\% poly(D-lactide). This nontoxic, biocompatible copolymer has an extensive history of use in a variety of medical applications such as suture materials, orthopedic, dental, ophthalmic, and craniofacial implants. Once implanted in vivo, over time, the copolymer chains degrade into water soluble fragments that are naturally found in the body (i.e., lactic acid) and are metabolized and eliminated through normal physiologic pathways. Landes et al studied 70\% poly(L-lactide) 30\% poly(D-lactide) copolymer degradation in human subjects in the maxillofacial region applications. $^{20}$ The copolymers decomposed reliably in patients within 24 months on average, leaving only extremely small granules that powder upon finger touch. Landes et al also noted fibrous capsule formation at around 3 months postimplantation. For the current application for the support of the nasal lateral wall, it is hypothesized that the eventual encapsulation of the implant and then replacement of the implant substrate with fibrocollagenous scar tissue may provide support to the lateral wall over time; however, the strength and quality of the scar, as well as the potential for a prolonged improvement that outlasts the mechanical integrity of the absorbable implant is yet to be determined. 
In summary, NVC attributed to a weak lateral cartilage is a common cause of nasal obstruction. However, due to complex surgical techniques for correcting NVC and associated cosmetic consequences, NVC frequently remains untreated. ${ }^{2}$ Hence, in this study, we present a first-in-human experience with a minimally invasive technique for supporting the lateral nasal wall with low cosmetic risk and using a widely used absorbable material with well-known safety profile.

\section{Conflict of Interest}

The authors declare no conflict of interests.

\section{Funding}

This study was supported with research funding from Spirox Inc., Menlo Park, CA. Dr. San Nicoló received research funding by Spirox Inc., Menlo Park, CA, and is consultant to Spirox. Prof. Sadick received and Dr. Bas received research funding from Spirox Inc., Menlo Park, CA.

\section{References}

1 Mink PJ. Physiologie der Obern Luftwege. Leipzig. Germany: Vogel; 1920

2 Becker SS, Dobratz EJ, Stowell N, Barker D, Park SS. Revision septoplasty: review of sources of persistent nasal obstruction. Am J Rhinol 2008;22(4):440-444

3 Most SP. Trends in functional rhinoplasty. Arch Facial Plast Surg 2008;10(6):410-413

4 Most SP. Comparing methods for repair of the external valve: one more step toward a unified view of lateral wall insufficiency. JAMA Facial Plast Surg 2015;17(5):345-346

5 Stewart MG, Smith TL, Weaver EM, et al. Outcomes after nasal septoplasty: results from the Nasal Obstruction Septoplasty Effectiveness (NOSE) study. Otolaryngol Head Neck Surg 2004; 130(3):283-290

6 Lavinsky-Wolff M, Camargo HL Jr, Barone CR, et al. Effect of turbinate surgery in rhinoseptoplasty on quality-of-life and acoustic rhinometry outcomes: a randomized clinical trial. Laryngoscope 2013;123(1):82-89

7 Sufyan A, Ziebarth M, Crousore N, Berguson T, Kokoska MS. Nasal batten grafts: are patients satisfied? Arch Facial Plast Surg 2012; 14(1):14-19

8 Murrell GL. Auricular cartilage grafts and nasal surgery. Laryngoscope 2004;114(12):2092-2102

9 Cochran CS, Ducic Y, DeFatta RJ. Restorative rhinoplasty in the aging patient. Laryngoscope 2007;117(5):803-807

10 Islam A, Arslan N, Felek SA, Celik H, Demirci M, Oguz H. Reconstruction of the internal nasal valve: modified splay graft technique with endonasal approach. Laryngoscope 2008;118(10): 1739-1743

11 Toriumi DM, Josen J, Weinberger M, Tardy ME Jr. Use of alar batten grafts for correction of nasal valve collapse. Arch Otolaryngol Head Neck Surg 1997;123(8):802-808

12 Akcam T, Friedman O, Cook TA. The effect on snoring of structural nasal valve dilatation with a butterfly graft. Arch Otolaryngol Head Neck Surg 2004;130(11):1313-1318

13 Winkler AA, Soler ZM, Leong PL, Murphy A, Wang TD, Cook TA Complications associated with alloplastic implants in rhinoplasty. Arch Facial Plast Surg 2012;14(6):437-441

14 Ramakrishnan JB, Danner CJ, Yee SW. The use of porous polyethylene implants to correct nasal valve collapse. Otolaryngol Head Neck Surg 2007;136(3):357-361

15 Berghaus A. Implants for reconstructive surgery of the nose and ears. GMS Curr Top Otorhinolaryngol Head Neck Surg 2007;6: Doc06

16 Khosh MM, Jen A, Honrado C, Pearlman SJ. Nasal valve reconstruction: experience in 53 consecutive patients. Arch Facial Plast Surg 2004;6(3):167-171

17 Stewart MG, Witsell DL, Smith TL, Weaver EM, Yueh B, Hannley MT. Development and validation of the Nasal Obstruction Symptom Evaluation (NOSE) scale. Otolaryngol Head Neck Surg 2004; 130(2):157-163

18 Lipan MJ, Most SP. Development of a severity classification system for subjective nasal obstruction. JAMA Facial Plast Surg 2013; 15(5):358-361

19 Rhee JS, Sullivan CD, Frank DO, Kimbell JS, Garcia GJ. A systematic review of patient-reported nasal obstruction scores: defining normative and symptomatic ranges in surgical patients. JAMA Facial Plast Surg 2014;16(3):219-225, quiz 232

20 Landes CA, Ballon A, Roth C. In-patient versus in vitro degradation of P(L/DL)LA and PLGA.J Biomed Mater Res B Appl Biomater 2006; 76(2):403-411 